\title{
BMJ Open Severe Asthma Toolkit: an online resource for multidisciplinary health professionals - needs assessment, development process and user analytics with survey feedback
}

Steven Maltby (10 , 1,2,3 Peter G Gibson, ${ }^{1,2,3,4}$ Helen K Reddel, ${ }^{1,5}$ Lorraine Smith, ${ }^{1,6}$ Peter A B Wark, ${ }^{1,2,3,4}$ Gregory G King, ${ }^{1,5}$ John W Upham, ${ }^{1,7}$ Vanessa L Clark, ${ }^{1,2,3}$ Mark Hew, ${ }^{8}$ Louisa Owens, ${ }^{9}$ Stephen Oo, ${ }^{10}$ Alan L James, ${ }^{1,11}$ Bruce Thompson, ${ }^{1,12}$ Guy B Marks, ${ }^{1,5,13}$ Vanessa M McDonald ${ }^{1,2,3,4}$

To cite: Maltby S, Gibson PG, Reddel HK, et al. Severe Asthma Toolkit: an online resource for multidisciplinary health professionals-needs assessment, development process and user analytics with survey feedback. BMJ Open 2020;10:e032877. doi:10.1136/ bmjopen-2019-032877

- Prepublication history and additional material for this paper are available online. To view these files, please visit the journal online (http://dx.doi org/10.1136/bmjopen-2019032877).

Received 11 July 2019 Revised 01 March 2020 Accepted 05 March 2020
D) Check for updates

(c) Author(s) (or their employer(s)) 2020. Re-use permitted under CC BY-NC. No commercial re-use. See rights and permissions. Published by BMJ.

For numbered affiliations see end of article.

\section{Correspondence to}

Professor Vanessa M McDonald; Vanessa.McDonald@newcastle. edu.au

\section{ABSTRACT}

Objectives Severe asthma imposes a significant burden on individuals, families and the healthcare system. New treatment and management approaches are emerging as effective options for severe asthma. Translating new knowledge to multidisciplinary healthcare professionals is a priority. We developed 'The Severe Asthma Toolkit' (https://toolkit.severeasthma.org.au) to increase awareness of severe asthma, provide evidence-based resources and support decisionmaking by healthcare providers.

Setting Roundtable discussions and a survey of Australians clinicians were conducted to determine clinician preferences, format and content for a severe asthma resource.

Participants A reference group from stakeholder and consumer bodies and severe asthma experts provided advice and feedback. A multidisciplinary team of international experts was engaged to develop content. Written content was based on up-to-date literature. Peer and editorial review were performed to finalise content and inform web design. Website design focused on user experience, navigation, engagement, interactivity and tailoring of content for a clinical audience.

Results A web-based resource was developed. Roundtable discussions and a needs assessment survey identified the need for dedicated severe asthma management resources to support skills training. The end-product, which launched 26 March 2018, includes an overview of severe asthma, diagnosis and assessment, management, medications, comorbidities, living with severe asthma, establishing a clinic, paediatrics/ adolescents and clinical resources. Analytics indicate access by users worldwide (32 169 users from 169 countries). User survey results $(\mathrm{n}=394)$ confirm access by the target audience ( $72 \%$ health professionals), who agreed the toolkit increased their knowledge $(73 \%)$ and confidence in managing severe asthma (66\%), and $75 \%$ are likely to use the resource in clinic.

Conclusions The Severe Asthma Toolkit is a unique, evidence-based internet resource to support healthcare

\section{Strengths and limitations of this study}

- Surveys of multidisciplinary healthcare professionals identified the need for an online resource dedicated to severe asthma.

- This report describes the development of a novel website resource designed to increase awareness of severe asthma, provide evidence-based resources and support healthcare professionals.

- Written and visual content was developed by an international team of experts and underwent detailed internal review and approvals.

- User feedback from a diverse range of stakeholders was incorporated to enhance website functionality and clinical utility.

- Additional research is required to demonstrate the effects of the resource on clinician practice and patient outcomes.

professionals providing optimal care for people with severe asthma. It is a comprehensive, accessible and independent resource developed by leading severe asthma experts to improve clinician knowledge and skills in severe asthma management.

\section{INTRODUCTION}

Asthma is a common disease, affecting over 330 million people worldwide. ${ }^{1}$ Over the last 30 years, there have been significant advances in asthma management both in terms of pharmacotherapy and self-management initiatives, resulting in improved outcomes for patients including reduced asthma mortality. However, approximately 3\%-10\% of people with asthma have severe treatmentrefractory disease, which fails to respond to standard therapies despite confirmation of diagnosis, management of common problems such as 
poor adherence and treatment of comorbidities, and outcomes for this population are poor. ${ }^{23}$ People with severe asthma experience significant and additional disease burden compared with individuals with mildto-moderate asthma, including ongoing uncontrolled symptoms, ${ }^{4}$ impaired quality of life,${ }^{56}$ frequent attacks, hospitalisations ${ }^{7}$ and early death. ${ }^{8}$ Severe asthma also places a substantial financial burden on affected individuals and society. ${ }^{9} 10$

With the recognition that severe asthma is different from mild-to-moderate disease, and has unique diagnostic, assessment and management requirements, new approaches to pharmacotherapy and disease management are emerging. ${ }^{8}$ These include an increasing number of novel treatment options for people with severe disease, such as monoclonal antibody therapies, which require measurement of biomarkers to identify those patients most likely to respond to therapies targeted to, at this stage, 'T2 high' asthma inflammatory pathways. ${ }^{11}$ While these treatments are revolutionising severe asthma management in terms of improved patient outcomes, their implementation into practice requires support; this could be in the form of new resources, skills and training for clinicians. ${ }^{12}$ Implementation of approaches to identify and manage asthma that is difficult to control because of treatable problems and comorbidities is needed. ${ }^{12}$ Furthermore, new paradigms for disease management are now proposed (eg, 'treatable traits'), ${ }^{13} 14$ and there is a need for ongoing development of disease and management paradigms. At present, our healthcare systems are ill-equipped to implement these approaches, ${ }^{15}$ and our multidisciplinary clinician training programmes have not yet been redesigned to facilitate training in these approaches. ${ }^{12}$ Furthermore, much of the clinical education available relating to asthma focuses on mildto-moderate disease, and this knowledge and training is often extrapolated and applied to patients with severe disease, for example, a stepwise escalation of antiinflammatory corticosteroid treatment that is appropriate for mild-to-moderate disease. This is inappropriate for severe asthma, which responds differently to treatment, impacts patients in different ways and requires a different approach to management (eg, targeted add-on therapy).

Severe asthma resources that have been developed have primarily been tailored for a patient audience, providing an overview of the topic rather than clinical guidance (eg, Asthma Australia, Asthma UK websites and the recently launched HealthTalk Australia severe asthma website). Specific educational and clinical resources for severe asthma are required for health professionals, as we previously identified in roundtable discussions by a panel of severe asthma experts. ${ }^{12}$ Educational resources to up-skill multidisciplinary clinicians and to support management where specialist care is limited (eg, rural and remote regions) are also needed. ${ }^{12}$

There is a growing use of online resources in health professional education. ${ }^{16}{ }^{17}$ E-learning approaches enhance and enable flexible learning. ${ }^{17} 18$ Website-based resources provide flexibility in the time and delivery of education and are ideally suited for the broad distribution and delivery of educational materials, ${ }^{17}{ }^{18}$ including to remote and rural settings. Online resources are also easily accessible in clinical settings, providing an opportunity for practical utility to inform practice. To our knowledge, no dedicated educational and training websites exist to inform the clinical management of severe asthma.

We proposed that a web-based resource designed for clinicians to increase awareness of severe asthma and to inform the management of severe asthma was required. We developed the Severe Asthma Toolkit website (https://toolkit.severeasthma.org.au) ${ }^{19}$ to address this need. Content is targeted at clinicians in primary and specialist care, in both private and public clinic settings. The Severe Asthma Toolkit is an educational resource providing up-to-date information on topics relevant to severe asthma and a practical, evidence-based guide to inform disease management. The purpose of this article is to describe the processes undertaken in: (1) assessing the need for a severe asthma resource, (2) informing the content and format, (3) the development process and (4) to report data on resource usage and user feedback.

\section{METHODS}

This project was initiated by the Translation Committee of the Centre of Excellence in Severe Asthma. ${ }^{20}$ The Centre of Excellence in Severe Asthma (https://www.severeasthma.org.au) is a network of Australian researchers funded by the Australian National Health and Medicine Research Council's (NHMRC) Centres of Research Excellence programme. The Centre of Excellence was funded to generate new knowledge in severe asthma, translate research evidence into policy and practice, develop novel resources and build clinical workforce capacity. Development of the Severe Asthma Toolkit was led by Centre of Excellence chief investigators, and all funding for the project was provided by the Centre of Excellence.

\section{Conception and project scoping}

In December 2015, we hosted a full day of roundtable discussions with multidisciplinary experts from a range of healthcare disciplines, stakeholder organisations and biopharmaceutical industry. ${ }^{12}$ The aim of this meeting was to identify knowledge gaps in severe asthma research and practice and to develop consensus on areas for improvement. $^{12}$ Key management gaps were identified, including limited awareness of severe asthma and limited access to high-quality multidisciplinary training programmes for clinicians treating patients with severe asthma; new resources to assist clinicians in severe asthma management were proposed. ${ }^{12}$ We then carried out a needs assessment survey during a Post-Graduate Workshop on Severe Asthma held at the 2017 Thoracic Society of Australia and New Zealand (TSANZ) Annual Scientific Meeting to determine demand for a dedicated severe 


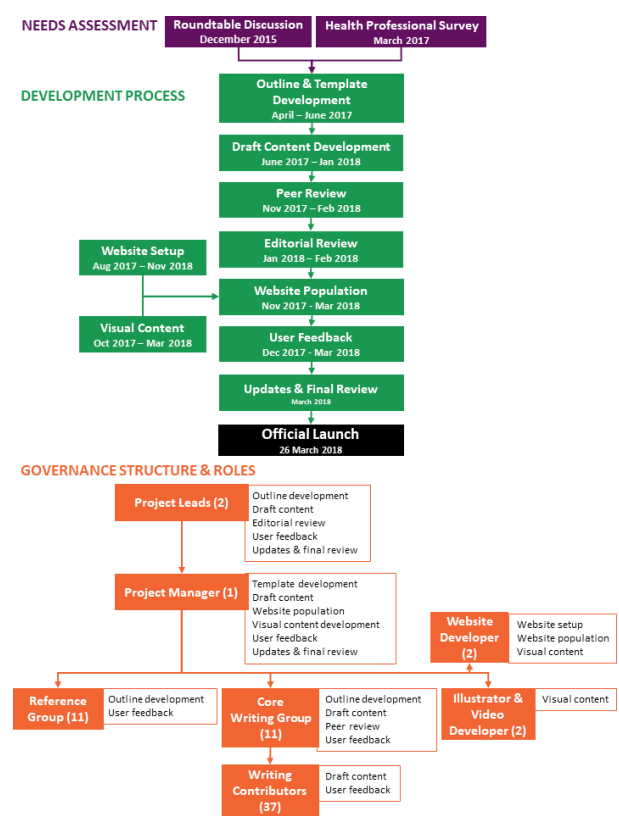

Figure 1 Overview of content development process and governance for the Severe Asthma Toolkit. A flowchart illustrating the key steps involved in development of the Severe Asthma Toolkit, the contributors involved and their areas of contribution. The number of contributors are indicated in brackets.

asthma resource and to inform the type and format of content.

\section{Patient and public involvement}

Content was codesigned with consumer representatives and end-users. A reference group that included a consumer advocate, a person with severe asthma and members of national consumer organisations, including Australia Australia, National Asthma Council Australia and the Lung Foundation of Australia was convened. Content was reviewed by representatives and amendments made to the content based on the feedback.

\section{Written content development}

An overview of the project development process and project governance is presented in figure 1 . The project was overseen by two expert severe asthma clinician researchers who led project development, advised and reviewed content and provided feedback and editorial sign-off prior to publication of any content (Peter G Gibson and Vanessa M McDonald). A reference group was established, consisting of representatives from peak stakeholder organisations and consumer groups (including representatives from Asthma Australia, TSANZ, National Asthma Council and Lung Foundation Australia) and multidisciplinary healthcare professionals to provide advice and feedback on drafted content and website design. The reference group also ensured that materials aligned with existing content developed by stakeholder organisations and facilitated endorsement by stakeholder organisations as relevant.
A writing committee involving a team of multidisciplinary clinicians and scientists with relevant expertise in severe asthma management was engaged to develop content. Core writing group members were assigned to lead the development of individual toolkit modules and finalised the outline, informed website strategy and drafted and reviewed content. Section leads recruited additional contributors with necessary expertise to draft subsection content with support from the project manager. Contributors included physicians, nurses, allied health professionals and scientists with a range of relevant expertise. Development was facilitated by a project manager (Steven Maltby) who collated initial outlines, developed template documents and integrated content based on feedback from clinician researchers, coordinated communications and provided progress updates. All written content was provided pro bono, and no authors had direct links to industry/pharmaceutical companies. A full list of contributors has been provided in online supplementary table 1 .

Written content was extensively referenced to peerreviewed literature, with hyperlinks to facilitate access to research evidence. Section leads performed peer review on modules that they were not involved in drafting. Additional editorial review was performed for consistency of message, style and format. Reviewers provided specific feedback on drafted content, identified gaps and redundant elements and highlighted text that could be converted into engaging visual content. All content was approved by section leads and the project co-leads prior to inclusion on the final website.

\section{Website and audiovisual content development}

A web designer (Hyperweb) was employed to develop logos, branding and build the website on a WordPress content management system. The website was designed to emphasise user experience, ease of navigation, graphics, engagement, interactivity, visual appeal and the amount, type and appropriateness of content. Images, videos, graphs and schematics were included to complement written content and clarify key messages. Visual content was developed in consultation with a web developer, videographer and illustrator. Patient videos were provided by content contributors, as acknowledged on the toolkit website, with written consent from the patients. Linked educational seminars were recorded as part of the Centre of Excellence severe asthma seminar series. Written consent was provided by participants to share seminar videos. Additional images were sourced from stock photography websites. Web content was optimised for viewing on mobile phones and tablets. Search engine optimisation was performed on a page-by-page basis to identify relevant search terms and optimise text content.

\section{Prelaunch user feedback}

Detailed feedback was sought from core writing and reference group members $(n=22)$ prior to website launch. Feedback was specifically sought on overall impression, user experience, visual elements, written content, usefulness 
of the resource and gaps before website launch. Feedback was incorporated into the final website version.

In addition to user feedback from contributors, potential users $(n=3)$ were recruited from the backgrounds of medicine, nursing and respiratory science prior to the website launch to provide feedback on website functionality. Users explored the website with minimal direction and provided verbal feedback, according to the "thinkaloud' technique. ${ }^{21}$ Users were then specifically asked about their experience, including overall impression, user experience, navigation, graphics, engagement, interactivity and the amount and type of content presented. Feedback was compiled to inform website design and revisions prior to launch.

Website usage statistics, including number of users/ page-views, user location, time spent on the website and navigation behaviour within the website were captured using Google Analytics. Video viewing statistics including number of views and total view time were captured by YouTube analytics.

A pop-up user feedback survey was integrated into the website. Respondents were asked about demographics, how they discovered the website, features they were seeking and feedback on content (questions and responses are included in online supplementary table 2).

\section{Data sharing statement}

Data relating to a needs assessment survey, user analytics and user feedback surveys are included below. No additional data are available from this study.

\section{RESULTS}

\section{Establishing the need}

The 2015 roundtable discussion, as previously reported, ${ }^{12}$ highlighted the need for new resources to inform severe asthma management. We conducted a needs assessment survey at the 2017 TSANZ Annual Meeting in Canberra (Australia), which included a range of healthcare professionals, including physicians, nurses and allied health professionals, with an interest in respiratory disease. Respondents were asked 'do you think there is a need for a new resource dedicated to severe asthma' and 'what formats would be useful to you?'. All respondents ( $n=24)$ indicated a need for a new resource dedicated to severe asthma, with an online/website format $(21 / 24 ; 88 \%)$ and interactive modules $(15 / 24 ; 63 \%)$ considered most useful. A minority of respondents also indicated that brochures $(6 / 24 ; 25 \%)$ and videos $(4 / 24 ; 17 \%)$ would be useful. Respondents prioritised practical checklists $(21 / 24 ; 88 \%)$, clinic protocols $(21 / 24 ; 88 \%)$, summary presentations $(18 / 24 ; 75 \%)$ and literature references $(15 / 24 ; 63 \%)$ as useful inclusions in the resource. Respondents also indicated content areas that would be useful (eg, dysfunctional breathing, checklists, questionnaires, information about monoclonal antibodies and comorbidities). Website format and contents were informed by these preferences.

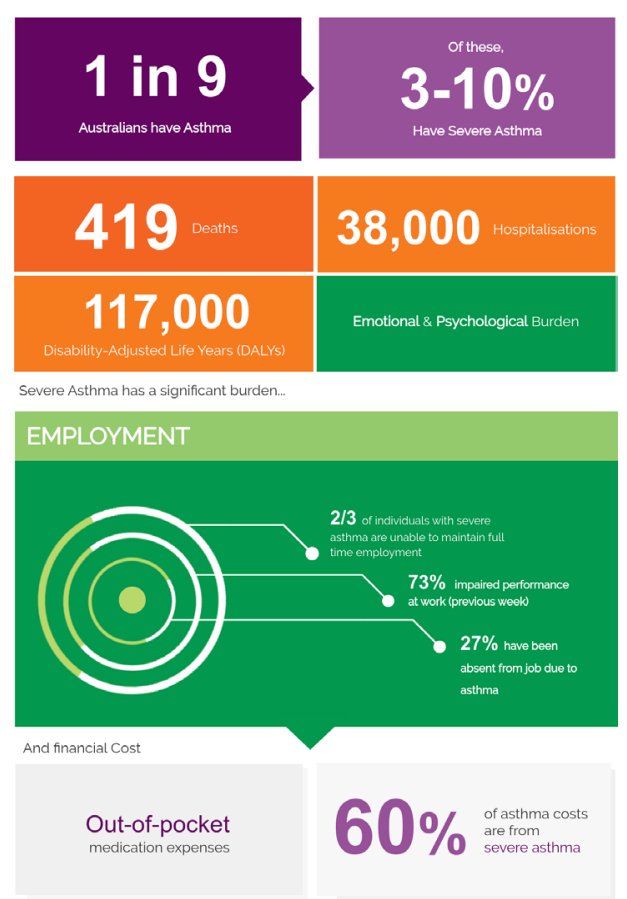

Figure 2 Representative figure from the Severe Asthma Toolkit. A schematic diagram outlining features of severe asthma prevalence and disease burden.

\section{Content development}

Content was developed in modules to allow users to work systematically through a single topic or to navigate directly to specific content. Each toolkit module consists of an overview page that supports navigation to subpages, which provide in-depth information on each topic. Images and schematics are featured throughout to clarify content and improve user experience (figure 2). A total of 36 videos were embedded in or accessible from the website at launch, including an overview of the toolkit, educational seminars and patient interviews. Modules and subpages are presented in table 1, along with a description of the content included. Each module focuses on a different topic relevant to severe asthma. A 'contact' page enables user feedback. A legal disclaimer is also included to clarify that the website should not be relied on by individual patients as a substitute for medical advice from healthcare professionals.

\section{Prelaunch user feedback}

Feedback obtained prelaunch from reference and writing group members and potential users was overall positive and provided some ideas for improvement. Reference and writing group members provided feedback from a range of perspectives, as these groups included representatives from stakeholder organisations, consumers and multidisciplinary healthcare professionals. Reviewers indicated the website was easy to navigate, had engaging and useful images and video content and provided educational content at an appropriate level. Feedback from consumer representatives on the reference group emphasised the importance of a patient-centred care perspective 
Table 1 Severe Asthma Toolkit content layout and description

\begin{tabular}{ll}
\hline Module & Description \\
\hline About the Severe Asthma Toolkit & An introduction to the resource outlining the identified need and included content areas. \\
What is severe asthma? & $\begin{array}{l}\text { Information on disease definitions, prevalence, disease burden, pathophysiology, } \\
\text { symptoms, asthma attacks and phenotypes. }\end{array}$
\end{tabular}

Diagnosis and assessment

Outlines the importance of confirming an asthma diagnosis and a practical stepwise approach for diagnosis and assessment, including considerations for the use of lung function assessments, questionnaires, allergy assessment, phenotyping, bronchoscopy, imaging and assessment of occupational asthma.

Management

Provides recommendations for an integrated, multidisciplinary approach, including content to support asthma education, development of written action plans, reviewing and reinforcing self-management skills, multidisciplinary approaches and referral.

Outlines the benefit from a targeted treatment approach, rather than the stepwise approach
used for mild-to-moderate disease. An overview of reliever, controller and add-on
therapies, including recommendations for the use of monoclonal antibodies and bronchial
thermoplasty.

Comorbidities

Highlights the importance of considering and systematically assessing comorbidities, which contribute to worsened severe asthma outcomes. Content covers pulmonary and extrapulmonary comorbidities, including allergic and non-allergic rhinitis, chronic rhinosinusitis, dysfunctional breathing, vocal cord dysfunction, chronic obstructive pulmonary disease, bronchiectasis, obstructive sleep apnoea, obesity, anxiety and depression, gastro-oesophageal reflux disease, osteoporosis and cardiovascular and metabolic disease.

$\begin{array}{ll}\text { Living with severe asthma } & \begin{array}{l}\text { A patient-centred view of the patient experience including recorded interviews, describing } \\ \text { daily symptom burden, mental and emotional health, intimacy and relationships, self- } \\ \text { management support, medication costs, experience of care and attacks and prognosis. }\end{array} \\ \text { Establishing a clinic } & \begin{array}{l}\text { Recognising the complexity of organising and delivering care from a dedicated severe } \\ \text { asthma clinic, content focusses on set-up, staffing and multidisciplinary team approach, } \\ \text { facilities, delivery approach, tailored referrals, evaluation, opportunities for training and } \\ \text { research, and barriers and hurdles. }\end{array} \\ \text { Paediatrics and adolescents } & \begin{array}{l}\text { Recognising additional considerations for different patient populations, specific content is } \\ \text { included for paediatrics and adolescents focused on management, assessment, alternative } \\ \text { diagnosis and comorbidities, psychosocial issues and medications. }\end{array} \\ \text { Specific populations } & \begin{array}{l}\text { Material specific for Indigenous Australians and Culturally and Linguistically Diverse } \\ \text { Backgrounds. }\end{array} \\ \text { Registries } & \begin{array}{l}\text { An overview of disease registries and specific details on enrolling patients in current } \\ \text { Australia severe asthma registries, including the Severe Asthma Web-based Database, } \\ \text { Australian Mepolizumab Registry and the Australian Xolair Registry. }\end{array} \\ \text { Resources } & \begin{array}{l}\text { A range of practical tools for use in clinical practice, including clinic recommendations, } \\ \text { resources for assessment and systematic and multidimensional assessment, guidance } \\ \text { for translation and implementation activities, seminar presentations, videos, links and key } \\ \text { references. }\end{array}\end{array}$

Module subheadings are indicated in italics.

throughout the toolkit, including the use of images with diversity in cultural backgrounds and clinical settings, and use of 'lay language' summaries, where possible. Potential users $(\mathrm{n}=3)$ provided feedback in interviews on website functionality and user experience, agreeing that the website was easy to navigate, engaging and educational. Examples of user quotes included 'well laid-out', 'helpful resource', 'useful format for broad range of audiences', 'good balance of graphics/text', 'written at an accessible level for multiple audiences' and 'the project is a bold endeavour, which is needed'. Suggested improvements were integrated into the final website design (eg, reference hyperlinks, improved patient-centred language and representative diversity in images).

The Severe Asthma Toolkit was made available online 23 March 2018 and officially launched on 26 March at the TSANZ Annual Scientific Meeting in Adelaide, Australia. From 23 March 2018 to 21 November 2019, 32169 total users accessed the website (42 454 sessions; 99369 page views) from 169 different countries. The most represented countries are shown in figure 3A. Returning users made up $13 \%$ of visits (4 182 visitors). Users accessed the site via organic searches (eg, Google; 69\%), direct links $(20 \%)$, referral from other sites $(9 \%)$ and social media 

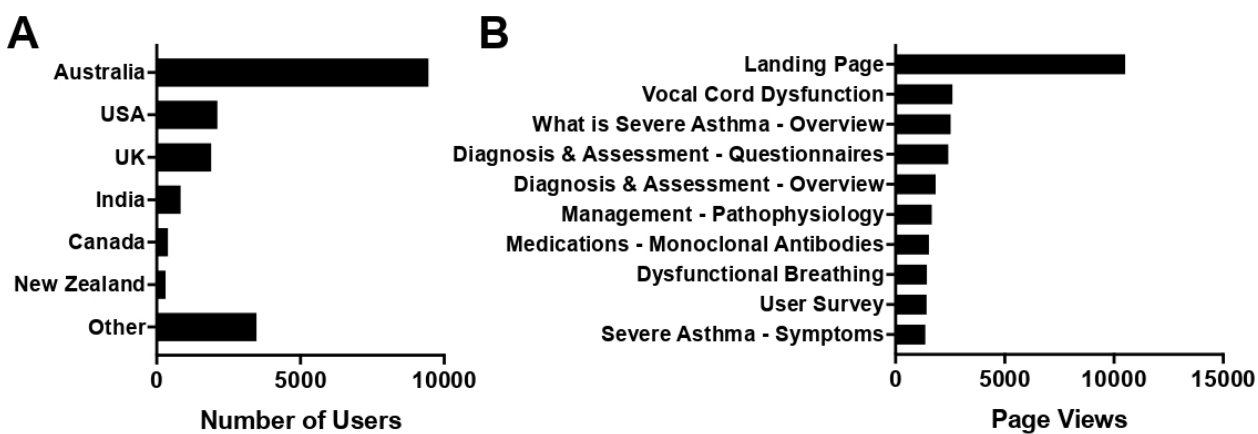

Figure 3 Severe Asthma Toolkit user access characteristics. Analytics data of: (A) user country and (B) total number of page views ( $n=32169$ users).

(eg, Twitter; 2\%). Users accessed the website using a range of technologies, including desktop $(62 \%)$, mobile phones $(32 \%)$ and tablets $(6 \%)$ and spent an average of 2 min $27 \mathrm{~s}$ on the website per session. The most accessed pages are shown in figure 3B. Over the same time period, linked and embedded videos were viewed 5500 times, with a total of over 460 hours of viewing time.

\section{Postlaunch user feedback}

During the period between 23 March 2018 and 21 November 2019, 394 website visitors completed the on-site user feedback survey. Most identified as health professionals $(285 / 394 ; 72 \%)$, working in a respiratory (147/394; 37\%) or non-respiratory field (138/394; 35\%; figure $4 \mathrm{~A})$. The remaining users included individuals with severe asthma or family members $(59 / 394 ; 15 \%)$, medical trainees $(9 / 394 ; 2 \%)$, two patient advocates or 'other' (39/394; 10\%). Respondents ages ranged: $29 \%$ were $45-54$ years $(114 / 391)$ and $26 \%$ were $55-64$ years of

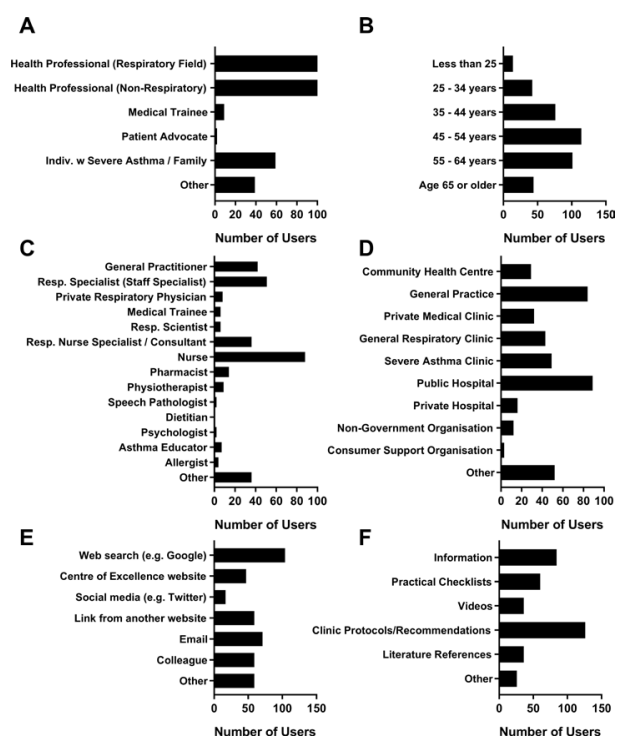

Figure 4 User feedback survey responses including demographics, website access and feedback. Users provided feedback on: (A) position description, (B) age group, (C) position title, (D) setting where they interact with patients with severe asthma, (E) how they found out about the Severe Asthma Toolkit and (F) most useful resources ( $n=394$ respondents). age $(101 / 391)$ being the most represented (figure 4B). Position titles for respondents that identified as health professionals are included in figure 4C. Of note, a large proportion of respondents were nursing and allied health professionals (52\%). The majority of respondents had some previous experience with severe asthma management $(338 / 376 ; 90 \%)$, either relatively inexperienced $(132 / 376 ; 35 \%)$, well experienced $(174 / 376 ; 46 \%)$ or expert $(32 / 376 ; 9 \%)$. Respondents interact with patients in a range of settings, shown in figure $4 \mathrm{D}$. Respondents who answered 'other' $(n=52)$, interacted with patients in schools/teaching settings $(n=13)$, home visits $(n=6)$ and a range of other venues.

Most survey respondents $(325 / 386 ; 84 \%)$ were visiting the website for the first time, while $50 / 386(13 \%)$ had visited several times and 11/386 (3\%) visit regularly. Respondents heard about the website from a range of sources shown in figure 4E. Users that responded 'other' predominantly found out about the website from editorials/newsletters $(\mathrm{n}=22)$ and conferences/seminars $(n=8)$. Respondents indicated they were seeking each of the toolkit topic areas, with most seeking 'Management' (82\%), 'Medications' (63\%), 'Diagnosis \& Assessment' $(62 \%)$ and 'Resources' (57\%). Respondents indicated clinic protocols/recommendations as the most useful resource on the site $(126 / 368 ; 34 \%)$, along with other resources as shown in figure $4 \mathrm{~F}$.

Most survey respondents $(205 / 281 ; 73 \%)$ agreed that the Severe Asthma Toolkit increased their knowledge of severe asthma (figure 5A). The majority also agreed that the toolkit improved their confidence in managing severe asthma (184/277; 66\%; figure 5B), and most agreed $(195 / 259 ; 75 \%)$ that they are likely to use this resource in the clinic (figure 5C).

\section{DISCUSSION}

This paper describes the development and user feedback of a purpose-built educational resource designed to increase awareness of severe asthma and to aid multidisciplinary clinicians in providing evidenced-based contemporary care for patients with severe asthma. Our previous work indicated that new resources were needed, ${ }^{12}$ and the Severe Asthma Toolkit was developed to address this 
A

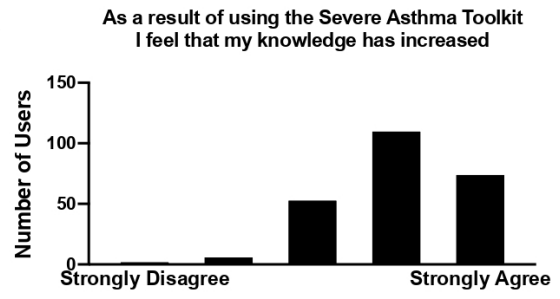

B As a result of using the Severe Asthma Toolkit I feel that my confidence in managing severe asthma has increased

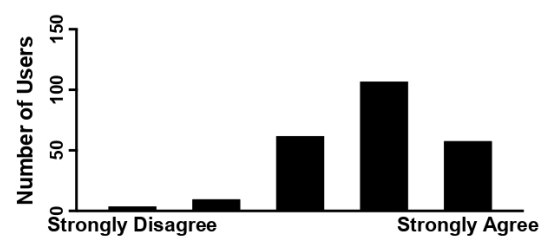

C I am likely to use the Severe Asthma Toolkit in the clinic

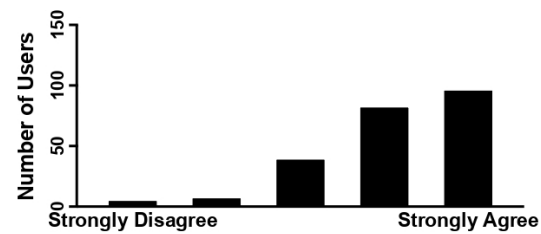

Figure 5 User survey feedback on resource utility. Users provided feedback on whether the Severe Asthma Toolkit increased their: (A) knowledge, (B) confidence in managing severe asthma and whether they are (C) likely to use the resource in the clinic ( $n=394$ survey respondents).

need. To our knowledge, this is the first peer-reviewed online severe asthma clinician web-based resource that has been developed and that has incorporated the identified needs of severe asthma clinicians.

Our initial needs assessment confirmed that a dedicated severe asthma resource was required. Living with severe asthma has a significant hidden burden, which is often under-recognised. ${ }^{22}$ The Severe Asthma Toolkit was developed in part to raise healthcare professionals awareness of this disease burden, incorporating patient interviews and content on the experience of living with severe asthma (eg, 'Living with Severe Asthma' module). Patients with severe asthma also have unique healthcare requirements in terms of self-management needs, treatment approaches and comorbidity management. ${ }^{8}$ These features present unique challenges for clinical management and healthcare systems. Knowledge transfer is a recognisable challenge in healthcare, ${ }^{23}$ and in this rapidly evolving area of severe asthma management, there was a clear need to develop a novel resource to address the needs of clinicians and to support education that integrates new management approaches. By presenting both educational and practical content, the Severe Asthma Toolkit addresses this need.

The value of this resource has been reinforced by the uptake and dissemination by stakeholder organisations to their respective healthcare professional networks (eg, Asthma Australia, TSANZ, National Asthma Council and Lung Foundation of Australia) and official recognition of the website as a TSANZ-endorsed training resource. The TSANZ is the only health peak body representing a range of professions (medical specialists, scientists, researchers, academics, nurses, physiotherapists and students) across various disciplines within respiratory medicine in Australia and New Zealand. The current membership of TSANZ is $\sim 1700$, which includes near complete reach to all Australian respiratory physicians and advanced trainees.

These organisations added links to the toolkit on their websites, disseminated details on the resource via email lists, advertised the research at training workshops and have cited the Severe Asthma Toolkit in their distribution materials (eg, National Asthma Council 'Monoclonal Antibody Therapy for Severe Asthma' information paper).

To summarise, new management approaches for severe asthma require access to healthcare resources. While new targeted therapy options are available that provide a personalised approach for patients, effective use of these treatments requires detailed phenotyping of clinical and immunological domains. ${ }^{11}$ Multidimensional assessment and management of comorbidities reduce exacerbations and improve asthma control and asthma-related quality of life. ${ }^{24}$ These approaches require recognition of comorbidities, access to multidisciplinary allied health professionals and effective coordination of care. Dedicated asthma services also improve healthcare use and patient outcomes. ${ }^{25}$ However, establishment of a severe asthma clinic is complex. The Severe Asthma Toolkit provides content to support users in each of these issues, with evidence-based practical recommendations (eg, modules on 'Asthma Phenotypes', 'Phenotyping', 'Multidimensional Assessment' and 'Establishing a Clinic') and educational infographics (accessible at https://toolkit. severeasthma.org.au/resources/infographics/).

Content development and website design were informed by a needs assessment, expert opinion and user feedback. The result is an online resource that is easy to navigate and contains a wide breadth of evidence-based content relevant to severe asthma in a single location. Google Analytics data identify that users seek and access content across all modules of the toolkit. Furthermore, the majority of survey respondents indicated that the toolkit increased their knowledge and confidence in delivering severe asthma management to patients. Importantly, three quarters of survey respondents indicated they will access the resource while seeing patients in the clinic.

Our needs assessment survey indicated that an online format would be most useful, among healthcare professional respondents. Online web formats can be readily accessible, free and provide rapid access to evidencebased medical information. The Bronchiectasis Toolbox ${ }^{26}$ and Australian Asthma Handbook ${ }^{27}$ are two examples in the respiratory field. Clinicians and medical trainees are increasingly accessing free online content to complement existing training programmes. ${ }^{28}$ User analytics data suggest that the Severe Asthma Toolkit is being accessed by this intended target audience ( $72 \%$ health professionals) 
across a range of health disciplines. For context, we note that the total membership of the Thoracic Society of Australia \& New Zealand (the peak professional respiratory body in these countries) is approximately 1700 total members. Thus, we propose that over 32000 total users accessing the Severe Asthma Toolkit represents significant access based on the intended target audience. The proportion of nursing and allied health professionals accessing the resources was particularly high. While the resource has been developed for a healthcare audience, the online format also provides access to patients, supporting additional education opportunities. We recognised that accessibility is important, allowing users to access from different settings (eg, clinic, home and office) and across broad geographical regions, including rural and remote regions. The utility and flexibility of this format is highlighted by users accessing content using a range of devices and from countries around the world, as well as survey respondents self-reporting that they would use the toolkit as a resource in clinic $(75 \%)$. Accordingly, the website is an accessible resource that provides both educational and clinical utility through point-of-care access to patient assessment tools, clinical guidelines and educational resources.

A further advantage of the online format is the ability to include updates. This is particularly relevant in relation to recent rapid advances in severe asthma treatments and management approaches. The website is a live document, which allows for the ongoing addition of content based on advances in the field. A 'last updated' note has been added to each web page to provide clarity on when content was posted. Development and inclusion of additional resources will be informed by user feedback to ensure that content remains up-to-date and aligns with user needs. All revised content will undergo approval by the section leads and project co-leads prior to publication.

The cost of creating web resources of this complexity can be a significant barrier. The Severe Asthma Toolkit was developed for a total cost of approximately $\$$ A20 000. This estimate includes costs for the web developer, illustrator, copyright permissions, graphic design, stock photography and search engine optimisation. The estimate does not include costs associated with the needs assessments, the in-kind support of the contributors, project manager or the project leaders, ongoing updates or writing costs. Much of these expenses were covered by existing Centre of Excellence in Severe Asthma activities, which is supported by NHMRC Centres of Research Excellence grant funding from 2014 to 2019. Centre of Excellence funding also supported social media activity (Twitter@SevereAsthmaCRE), which was used to increase awareness of the Severe Asthma Toolkit and its resources. All contributors provided written content and review pro bono. Development of this resource would not have been possible without their contributions. We do note that additional features were considered and not included in the resource due to budgetary constraints (eg, functional online forums with question-and-answer capability, which would have required dedicated ongoing staffing). Basic ongoing maintenance of the website (eg, domain registration, web hosting servers and basic software updates) are anticipated to be quite minimal (\$A1-2000/ year), although dependent on existing staff or volunteer contributions. Development of additional resources and extensive updates will be dependent on support from specific funding (eg, educational grants) or infrastructure funding. This could include modules targeted specifically for a consumer/patient audience, with collaborative input from relevant stakeholder organisations. We note that a HealthTalk Australia Severe Asthma website (https://healthtalkaustralia.org/severe-asthma/overview/) was recently launched by Centre of Excellence in Severe Asthma Investigators, which presents interviews of people living with severe asthma for a public audience.

Our goals for development of this resource were to produce a web-based resource that would provide up-todate and disease-specific advice for severe asthma clinicians. This has been achieved. In terms of our other goals, the results are promising. These aspects included promoting awareness of severe asthma; survey respondents indicated that the resource increased knowledge (73\% of respondents) and confidence $(66 \%)$ in severe asthma management and provides a resource that clinicians say they will access while in the clinics seeing patients $(75 \%)$. The toolkit has been accessed by health professionals engaged in severe asthma (72\% of respondents) worldwide in 169 different countries, with approximately $13 \%-16 \%$ of repeat user traffic. We do note that survey respondents represent a relatively small proportion of all users $(394 / 32169=1.2 \%)$, and caution should be used in extrapolating these data to the total user population. While these data are encouraging, we recognise that dissemination is only part of the translation process to bring about clinical practice change and improve patient outcomes. Further activities are required to translate this educational resource to support practice change, and additional assessment will be required to determine the reach of the Severe Asthma Toolkit and its effects on outcomes. Additional and ongoing work is now required to increase and maintain user traffic, including collaboration with stakeholder organisations, linking from contributor web pages, social media campaigns (eg, via the Centre of Excellence's Twitter account @SevereAsthmaCRE), workshops and professional conferences. The broad expertise of contributors and active engagement with health professionals, stakeholders and consumers throughout this process were critical to the planning, development and current level of dissemination of this website. This process was invaluable to identifying the specific needs of our target population and is highly recommended for any groups looking to develop a similar resource in their area. We identified several limitations that we would address if we were to develop a new resource. Earlier engagement with illustrators and graphic designers would have been useful to support further integration of visual and interactive elements. Furthermore, development of informative 
infographics and handouts summarising evidence from the toolkit may be valuable resources to support translation of evidence into clinical care. While stakeholders were actively engaged early in the process to provide input, a more thorough communications plan to support dissemination of the resource would have been beneficial. We are continuing to work on these aspects. This includes the development of additional resources, such as clinical recommendation documents, infographics, interactive case studies and additional content (eg, smoking cessation and oral corticosteroid treatment). To maximise the benefit of this resource, we are continuing to engage with other health professionals, including those with limited experience in severe asthma management in Australia and worldwide.

\section{CONCLUSION}

The Severe Asthma Toolkit was developed in response to identified unmet needs to increase awareness of severe asthma and support education and training of severe asthma multidisciplinary clinicians. The resource was developed with input from a range of stakeholders and informed by user feedback. The website has been accessed by users worldwide. Ongoing evaluation and updates to the content will ensure that content remains up-to-date and practical. We note that the development of the Severe Asthma Toolkit is only one aspect of the required implementation approach necessary to support healthcare professional awareness and training for severe asthma. A concerted effort is now required by all stakeholders to implement existing evidence and resources (including the Severe Asthma Toolkit) to improve clinical practice and provide optimal care for people with severe asthma.

\section{Author affiliations}

${ }^{1}$ NHMRC Centre of Excellence in Severe Asthma, New Lambton, New South Wales, Australia

${ }^{2}$ Priority Research Centre for Healthy Lungs, University of Newcastle, Callaghan, New South Wales, Australia

${ }^{3}$ Hunter Medical Research Institute, New Lambton Heights, New South Wales, Australia

${ }^{4}$ Department of Respiratory and Sleep Medicine, John Hunter Hospital, Newcastle, New South Wales, Australia

${ }^{5}$ Woolcock Institute of Medical Research, University of Sydney, Sydney, New South Wales, Australia

${ }^{6}$ School of Pharmacy, University of Sydney Faculty of Pharmacy, Sydney, New South Wales, Australia

${ }^{7}$ Department of Respiratory Medicine, Princess Alexandra Hospital TRI, South Brisbane, Queensland, Australia

${ }^{8}$ Department of Allergy, Immunology \& Respiratory Medicine, Alfred Hospital, Melbourne, Victoria, Australia

${ }^{9}$ Sydney Children's Hospital, Sydney, New South Wales, Australia

${ }^{10}$ Princess Margaret Hospital, Fiona Stanley Hospital and University of Western

Australia, Perth, New South Wales, Australia

${ }^{11}$ Department of Pulmonary Physiology and Sleep Medicine, Sir Charles Gairdner

Hospital, Nedlands, Western Australia, Australia

${ }^{12}$ Alfred Health, Melbourne, Victoria, Australia

${ }^{13}$ South Western Sydney Clinical School UNSW, Sydney, New South Wales, Australia

Twitter Steven Maltby @severeasthmaCRE and Vanessa M McDonald @nessmcd
Acknowledgements VMM and PGG co-led the development of the Severe Asthma Toolkit; SM served as project manager, and other authors were members of the Core Writing Group or Centre of Excellence management group. SM and VMM drafted the initial manuscript, and all authors reviewed and approved the manuscript prior to submission. The authors would like to thank the contributors who provided content and feedback for the Severe Asthma Toolkit. A complete list of contributors is available on the Severe Asthma Toolkit website https://toolkit. severeasthma.org.au/contributors/.

Contributors PGG and VMM initially conceived of the project and co-led development, with feedback from all authors. SM served as project manager. PGG, HKR, LS, PW, GGK, JWU, VLC, MH, LO, S0 and VMM acted as core writing leads, overseeing development and editorial review of individual website sections. ALJ, BT and GBM drafted or provided editorial review of written content. PGG and VMM provided overall editorial approval. SM and VMM drafted the initial manuscript, and all authors provided critical feedback and approved the final version for publication.

Funding Funding was provided through the National Health and Medical Research Council (NHMRC) Centres of Research Excellence programme (APP1078579)

Competing interests PGG holds an National Health and Medicine Research Council (NHMRC) Practitioner Fellowship, has participated in educational symposia funded by AstraZeneca, Boehringer Ingelheim, GlaxoSmithKline and Novartis, and has participated in studies funded by GlaxoSmithKline and AstraZeneca. HKR or her institute has received honoraria for providing independent medical education at symposia funded by AstraZeneca, GlaxoSmithKline, Novartis, Teva, Mundipharma and Boehringer Ingelheim; for data safety monitoring boards and/or advisory boards for AstraZeneca, GlaxoSmithKline, Merck, Novartis, Boehringer Ingelheim and for consulting for AstraZeneca and GlaxoSmithKline. Her institute has received independent research grants from AstraZeneca and GlaxoSmithKline for her research. PABW or his institute has received honoraria for providing independent medical education at symposia funded by AstraZeneca, GlaxoSmithKline, Novartis, Mundipharma, Boehringer Ingelheim and Vertex; for data safety monitoring boards and/or advisory boards for AstraZeneca, GlaxoSmithKline, Novartis, Boehringer Ingelheim, and Sanofi and for consulting for AstraZeneca and GlaxoSmithKline. His institute has received independent research grants from AstraZeneca GlaxoSmithKline Novartis and Vertex for his research. GGK and his research group have received consultancy fees for talks, chairmanship, advisory boards, sponsorship of conferences from AstraZeneca, Boehringer Ingelheim, GlaxoSmithKline, Menarini, MundiPharma, Novartis and Teva Pharma; unrestricted research grants from NHMRC, Boehringer Ingelheim, GlaxoSmithKline, Menarini, MundiPharma, philanthropic societies and Sydney University. JWU reports grants, speaker fees and consulting fees from AstraZeneca, GSK, Novartis, Boehringer Ingelheim and Menarini, but these are not directly related to the submitted manuscript. MH declares grants and other advisory board fees (made to his institutional employer Alfred Health) from AstraZeneca, GlaxoSmithKline, Novartis and Seqirus, for unrelated projects. LO declares participation on advisory boards for Boehringer Ingelheim. VMM is supported by an NHMRC TRIP fellowship, has participated in educational symposia funded by GlaxoSmithKline, AstraZeneca and Menarini and has participated advisory boards for GlaxoSmithKline, Novartis, AstraZeneca and Menarini. All other authors have no declared conflicts of interest.

Patient and public involvement Patients and/or the public were involved in the design, or conduct, or reporting, or dissemination plans of this research. Refer to the Methods section for further details.

Patient consent for publication Not required.

Ethics approval The pop-up user feedback survey was integrated into the website with approval from the Hunter New England Human Research Ethics committee (AU201805-12).

Provenance and peer review Not commissioned; externally peer reviewed.

Data availability statement Data relating to a needs assessment survey, user analytics and user feedback surveys are included in the manuscript. No additional data are available from this study.

Open access This is an open access article distributed in accordance with the Creative Commons Attribution Non Commercial (CC BY-NC 4.0) license, which permits others to distribute, remix, adapt, build upon this work non-commercially, and license their derivative works on different terms, provided the original work is properly cited, appropriate credit is given, any changes made indicated, and the use is non-commercial. See: http://creativecommons.org/licenses/by-nc/4.0/.

Steven Maltby http://orcid.org/0000-0003-1240-5964 


\section{REFERENCES}

1 Global-Asthma-Network. The global asthma report 2018. Auckland, New Zealand, 2018.

2 Hekking P-PW, Wener RR, Amelink M, et al. The prevalence of severe refractory asthma. J Allergy Clin Immunol 2015;135:896-902.

3 Chung KF, Wenzel SE, Brozek JL, et al. International ERS/ATS guidelines on definition, evaluation and treatment of severe asthma. Eur Respir J 2014;43:343-73.

4 Reddel HK, Sawyer SM, Everett PW, et al. Asthma control in Australia: a cross-sectional web-based survey in a nationally representative population. Med J Aust 2015;202:492-6.

5 Foster JM, McDonald VM, Guo M, et al. "I have lost in every facet of my life": the hidden burden of severe asthma. Eur Respir $J$ 2017;50:1700765.

6 McDonald VM, Hiles SA, Jones KA, et al. Health-Related quality of life burden in severe asthma. Med J Aust 2018;209:S28-33.

7 Poulos LM, Correll PK, Toelle BG, et al. Lung disease in Australia: prepared for lung Foundation Australia. Sydney, NSW: Woolcock Institute of Medical Research, 2014.

8 Pavord ID, Beasley R, Agusti A, et al. After asthma: redefining airways diseases. Lancet 2018;391:350-400.

9 Sweeney J, Brightling CE, Menzies-Gow A, et al. Clinical management and outcome of refractory asthma in the UK from the British thoracic Society difficult asthma registry. Thorax 2012;67:754-6.

10 Sadatsafavi M, Lynd L, Marra C, et al. Direct health care costs associated with asthma in British Columbia. Can Respir $J$ 2010;17:74-80.

11 Grainge CL, Maltby S, Gibson PG, et al. Targeted therapeutics for severe refractory asthma: monoclonal antibodies. Expert Rev Clin Pharmacol 2016;9:927-41.

12 McDonald VM, Maltby S, Reddel HK, et al. Severe asthma: current management, targeted therapies and future directions-A roundtable report. Respirology 2017;22:53-60.

13 Agusti A, Bel E, Thomas M, et al. Treatable traits: toward precision medicine of chronic airway diseases. Eur Respir J 2016;47:410-9.

14 Agustí A, Bafadhel M, Beasley R, et al. Precision medicine in airway diseases: moving to clinical practice. Eur Respir J 2017;50:1701655.
15 Gibson PG, McDonald VM, Marks GB. Asthma in older adults. Lancet 2010;376:803-13.

16 Ellaway R, Masters K. AMEE guide 32: e-learning in medical education Part 1: learning, teaching and assessment. Med Teach 2008;30:455-73.

17 Sinclair PM, Kable A, Levett-Jones T, et al. The effectiveness of Internet-based e-learning on clinician behaviour and patient outcomes: a systematic review. Int J Nurs Stud 2016;57:70-81.

18 Fordis M, King JE, Ballantyne CM, et al. Comparison of the instructional efficacy of Internet-based CME with live interactive CME workshops: a randomized controlled trial. JAMA 2005;294:1043-51.

19 The severe asthma toolkit: centre of excellence in severe asthma, 2018. Available: https://toolkit.severeasthma.org.au Archived at www.webcitation.org/77iE6qoR7 [Accessed 18 Apr 2019].

20 Centre of excellence in severe asthma Homepage: centre of excellence in severe asthma, 2016. Available: https://www. severeasthma.org.au Archived at http://www.webcitation.org/ 77iDoBEri[Accessed 18 Apr 2019].

21 Davison GC, Vogel RS, Coffman SG. Think-aloud approaches to cognitive assessment and the articulated thoughts in simulated situations paradigm. J Consult Clin Psychol 1997;65:950-8.

22 Foster JM, McDonald VM, Guo M, et al. "I have lost in every facet of my life": the hidden burden of severe asthma. Eur Respir $J$ 2017;50:1700765.

23 Kitson A, Brook A, Harvey G, et al. Using complexity and network concepts to inform healthcare knowledge translation. Int $\mathrm{J}$ Health Policy Manag 2017;7:231-43.

24 Clark VL, Gibson PG, Genn G, et al. Multidimensional assessment of severe asthma: a systematic review and meta-analysis. Respirology 2017;22:1262-75.

25 Gibeon D, Heaney LG, Brightling CE, et al. Dedicated severe asthma services improve health-care use and quality of life. Chest 2015;148:870-6.

26 Nicolson $\mathrm{CH}$, Holland AE, Lee AL. The bronchiectasis Toolbox-A comprehensive website for the management of people with bronchiectasis. Med Sci 2017;5:13.

27 NAC. Australian asthma Handbook. V1.2, 2016.

28 Purdy E, Thoma B, Bednarczyk J, et al. The use of free online educational resources by Canadian emergency medicine residents and program directors. CJEM 2015;17:101-6. 